and $\mathrm{C}$ virus infection, genital herpes, amoebiasis, giardiasis, and condyloma, were recorded.

Results A total of 230 MSM completed the interviews from November 2017 through October 2018. The median age was 35 years (range: 18-70). MSM at the high risk was found in 185 (80.4\%). MSM who knew PrEP was only 61.3\%. Positive willingness of PrEP was observed in 64.8\%. MSM who answered "No definitely" were 21 among 185 high risk MSM. Their main negative reasons were "I think my HIV acquisition risk is low" in $12 \mathrm{MSM}$ and "I don't like to take medicine" in 8 . Histories of STI therapy within 1 year were found in 81 MSM (35.2\%). Among them, 27 (33.3\%) had still the negative willingness. There was no correlation of the positive willingness and history of STIs $(p=0.66)$.

Conclusion Still one third of MSM in our cohort lacked the proper knowledge of PrEP. Especially it is important to allow themselves to assess their own risk of HIV infection.

Disclosure No significant relationships.

\section{P557 "FLUX NZ": AN ONLINE NATIONAL COHORT INVESTIGATING HIV, STI AND DRUG-RELATED PRACTICES AMONG NEW ZEALAND GAY AND BISEXUAL MEN}

${ }^{1}$ Peter Saxton*, ${ }^{2}$ Mohamed Hammoud, ${ }^{3}$ Samuel Andrews, ${ }^{4}$ David Newcombe, ${ }^{5}$ Anthony Walton, ${ }^{5}$ Seb Stewart, ${ }^{5}$ Ricky Te Akau, ${ }^{6}$ Mark Fisher, ${ }^{7}$ Kathryn Leafe, ${ }^{8}$ Carl Greenwood, ${ }^{9} \mathrm{~J}$ Green, ${ }^{2}$ Garrett Prestage. ${ }^{1}$ University of Auckland, School of Population Health, Auckland, New Zealand; ' University of New South Wales, Kirby Centre, Sydney, Australia; ${ }^{3}$ New Zealand Drug Foundation, Wellington, New Zealand; ${ }^{4}$ University of Auckland, Centre for Addiction Research (CFAR), Auckland, New Zealand; ${ }^{5}$ New Zealand AIDS Foundation, Auckland, New Zealand; ${ }^{6}$ Body Positive, Auckland, New Zealand; ${ }^{7}$ New Zealand Needle Exchange Programme, Christchurch, New Zealand; ${ }^{8}$ Drugs Health and Development Project, Wellington, New Zealand; ${ }^{9} T e$ Whariki Takapou, Waikato, New Zealand

\subsection{6/sextrans-2019-sti.631}

Background HIV pre-exposure prophylaxis (PrEP) and chemsex present both opportunities and challenges for sexual health improvement among gay and bisexual men (GBM). However, there is scant evidence to inform responses in New Zealand (NZ), notably prevalence, uptake and cessation of behaviours over time. We sought a better understanding by recruiting a diverse national cohort of GBM.

Methods Flux NZ is a national online prospective observational study investigating wellbeing among GBM in NZ. The protocol, questionnaire and data management system were based on a concurrent Australian study. Recruitment was predominantly via social media and community partners. Eligible participants were GBM living in NZ. We present baseline descriptions of PrEP, STI and illicit drug-related data among the cohort to date.

Results The 315 participants are broadly reflective of the target population $(10.8 \%$ identified as indigenous Maori, 59\% lived outside Auckland the largest city, mean age 33.6 years (SD 15.6), 8.3\% diagnosed HIV-positive). Among HIV-negative participants, 50\% had heard "a lot" and 44\% "a little" about PrEP; 18.2\% were currently using PrEP. 57\% of all participants had tested for STIs in the past 6 months; 7\%, 5.7\% and 3.2\% were diagnosed with gonorrhoea, chlamydia and syphilis respectively. Drug use in the past 6 months was $37.5 \%$ cannabis, $35.6 \%$ amyl, 17.5\% MDMA, 5.1\% methamphetamine, 4.8\% LSD,
4.4\% cocaine, 3.2\% GHB, 2.5\% amphetamine, 1.0\% ketamine, $0.3 \%$ synthetic cannabis, $0 \%$ mephedrone, $0 \%$ heroin, and $1.9 \%$ other hallucinogens/psychedelics. "Any" drug use was $57.8 \%$ in the past 6 months and $78.7 \%$ ever. Of those using "party drugs" (MDMA, amphetamine, cocaine, methamphetamine, GHB, ketamine, LSD) recently, 29.2\% had done so to enhance sex, the majority "once" (4.7\%) or "a few times" (15.1\%).

Conclusion The Flux online cohort design effectively recruits GBM in NZ. This will enable comparative research of the impact of HIV, STI and drug harm reduction policies in the two countries.

Disclosure No significant relationships.

\section{P558 SEXUAL HEALTH, SYNDEMICS AND ASSETS AMONG MEN WHO HAVE SEX WITH MEN: SECONDARY ANALYSIS OF MULTI-NATIONAL SURVEYS}

${ }^{1}$ Lisa Mcdaid, ${ }^{1}$ Paul Flowers, ${ }^{2}$ Olivier Ferlatte, ${ }^{3}$ Kareena Mcaloney-Kocaman, ${ }^{4}$ Mark Gilbert, ${ }^{3}$ Jamie Frankis. 'University of Glasgow, MRC/CSO Social and Public Health Sciences Unit, Glasgow, UK; ${ }^{2} B C$ Centre for Substance Use, Vancouver, Canada; ${ }^{3}$ Glasgow Caledonian University, Glasgow, UK; ${ }^{4} B C$ Centre for Disease Control, Clinical Prevention Services, Vancouver, Canada

\subsection{6/sextrans-2019-sti.632}

Background Globally, gay, bisexual and other men who have sex with men (GBMSM) experience an increased burden of poor sexual, mental and physical health. Syndemics theory provides a framework to understand these interrelated health problems and how to intervene.

Methods Comparative quantitative secondary analysis of syndemic-related data are presented from two international, online, cross-sectional surveys: SMMASH2 in Scotland, Wales, Northern Ireland and the Republic of Ireland $(\mathrm{N}=3220)$; and Sex Now in Canada $(\mathrm{N}=7872)$.

Results In both studies negative sexual, mental and physical health outcomes were clustered, providing evidence of the syndemic (SMMASH2 O/E Ratio=1.32, 95\% CI 1.25-1.40; Sex Now O/E Ratio=1.59, 95\% CI 1.45-1.73). There were differences between the studies in the variables that were associated with experience of the syndemic. In Sex Now we found experience of the syndemic was associated with worries about sexuality-related stigma (AOR 1.87, 95\% CI 1.23-1.54) and experience of discrimination $(\mathrm{AOR}=1.83$, 95\% CI 1.60 2.10). Equally, some community assets appeared to have a protective effect on the experience of syndemics. In Sex Now, aspirations were significant; e.g., being unlikely to achieve quality of life significantly associated with increased odds of experiencing the syndemic $(\mathrm{AOR}=1.89,95 \%$ CI 1.63-2.19), while measures of community engagement were not significant. In SMMASH2, sense of coherence - a measure of resilience - was significant (AOR $=0.98,95 \%$ CI 0.96-0.99), indicating that higher sense of coherence was associated with decreased odds of experiencing the syndemic.

Conclusion These results present an important step forward in our understanding of syndemics. They provide new insights into how to intervene to reduce the interrelated burden of poor sexual, mental and physical health among GBMSM and point to a theoretical mechanism through which assets-based approaches to health improvement could function.

Disclosure No significant relationships. 